\title{
VAC.02 - Genetic basis for yellow fever vaccine-associated viscerotropic disease (YEL-AVD): a preliminary report
}

Emersom Cicilini Mesquita ${ }^{1 \star}$; Deborah Araújo da Conceição ${ }^{1}$; Patrícia Mouta Nunes de Oliveira ${ }^{1}$; Tamiris Azamor da Costa Barros'; Qian Zhang²; Nicholas Hernandez²; Emmanuelle Jouanguy; Jean-Laurent Casanova ${ }^{2}$; Maria de Lourdes de Sousa Maia ${ }^{1}$; Reinaldo de Menezes Martins ${ }^{1}$ (In memoriam).

1Fiocruz/Bio-Manguinhos;

2The Rockefeller University;

3Fiocruz - Fundação Oswaldo Cruz;

Introduction: Despite generally considered as a safe vaccine, yellow fever vaccine (YFV) can rarely be associated with severe adverse reactions including yellow fever vaccine-associated viscerotropic disease (YEL-AVD). Although the mechanisms behind YEL-AVD cases remains elusive, its occurrence among family members suggests that genetic defects may play a role. Here, we report the investigative strategy and preliminary reports from a national-based, phase IV study, aimed at elucidating the mechanisms behind YEL-AVD.

Objective: To elucidate the mechanisms behind yellow fever vaccine-associated viscerotropic disease (YEL-AVD).

Methodology: This is an observational study. The study was approved by Evandro Chagas National Institute of Infectious Disease's ethic review board (CAAE 60575716.2.0000.5262). YEL-AVD cases were defined as follows: At least two organ dysfunctions within 30 days post YF vaccination, PLUS evidence of vaccine strain YFV (RT-PCR $\geq 3 \log 10 \mathrm{PFU} / \mathrm{mL}$ at any time point OR more than 14 days from symptoms onset OR culture/immunohistochemistry from tissue samples), PLUS negative investigation for dengue, leptospirosis, zika and hepatitis A virus. Survivors from YEL-AVD were identified by Bio-Manguinhos's Clinical Trials Unit (ASCLIN), together with local and national authorities from Brazil's National Immunization Program (NIP). Blood and saliva samples were collected from all participants, including family members. Skin biopsies were collected only for YEL-AVD survivors. Whole Exome Sequencing (WES) was performed in the patients and their relatives. cDNA sequencing and Western Blot (WB) analysis were done on cells from patients and healthy donors.

Results: Overall, a total of 48 individuals were included in the study (3 YEL-AVD survivors and 44 family members). P1 was a 12 years old girl, who presented renal, hepatic, hematologic, and respiratory dysfunctions. She was admitted to an intensive care unit (ICU) 10 days after YF vaccination and discharged 22 days after that. P2 was a 14 years old girl that presented hepatic and hematological dysfunctions. She was hospitalized 3 days after YF vaccination and discharged 14 days after that. P3 was a 29 years old man who presented hepatic, renal, and neurological dysfunctions. He was hospitalized 10 days after YF vaccination and discharged 8 days after that. Importantly, none of those patients had any traditional risk factor for YELAVD. P2 had a sister who presented a fatal case of YEL-AVD. P3 had two brothers that died a few days after YF vaccination.

Conclusion: We report the first genetic cause of YFV-AVD in P1. Future inclusions are necessary in order to corroborate our initial findings. Identifying genetic markers for YELAVD can lead to a point-of-care technology destined to prevent those rare events.

Keywords: Yellow fever vaccine; Yellow fever vaccine-associated viscerotropic disease; Genetic basis 